\title{
Artículos
}

\section{Los nuevos esquemas de Derecho y las implicaciones de Clasificación}

Roberto Abell B.

Investigador del CUIB/UNAM

\section{RESUMEN}

Du ran te los pri me ros se sen ta años de este si glo, la Bi blio te ca del Con gre so se de mo ró en ela bo rar una cla si fi ca ción para el de re cho, debi do a que las bi blio te cas an gloame ri ca nas no tu vie ron la ne ce si dad de una, por que la ma yo ría de los li bros de esa dis ci pli na fue ron publicados en series de casos que únicamente requirieron clasificación por autor. Esta larga demora significó que las bibliotecas mexicanas desarrollaran sus propias clasificaciones para su material jurídico.

Por cier to, al ini cio de 1969 y du ran te los años se ten ta la Bi blio te ca del Con gre so pu bli có al gu nos es que mas para el de re cho an gloamerica no. Sin em bar go, has ta los años ochen ta apa re cie ron los es que mas para el de re cho e u ro peo y la ti no ame ri ca no, el pri me ro fue parael de re cho ale mán y lue go para el de re cho fran cés, comple men ta dos para las cla sifi ca cio nes re gio na les delde re chola ti no ame rica noy eut ropeo con las subclases, KDZ, KG-KH y KJ-KKZ respectivamente.

Des pués de re sú me nes bre ves de los es que mas para el de re cho in ter na cio nal, subcla seJX y del de re cho en ge ne ral, sub cla se K, se in trodu je ron las cla sifica cio nes re gio na les para el de re cho eu ro peo y la ti no ame rica no. Es tos es tán re pre sen ta dos con ta blas, de prin ci pio a fin, para el de re cho re gio nal y lue go las de ju ris dic cio nes na cio na les con sus sub di vi sio nes parael de re cholocal. Se de talla la apli ca ción de estas tablas en referencias con el derecho latinoamericano.

Aho ra que la Biblio te ca del Con gre so ha ela bo ra do los es que mas de las áreas del de re cho más im por tan tes para las bi blio te cas me xi canas, existe el problema de su aplicación.

\section{ABSTRACT}

For the first sixty years of the pre sent cen tury, the Li brary of Con gress de la yed the pu blication of a clas si fi ca tion for law owing to the fact that Anglo-Americanlibraries felt no need for one what with law book published in series of case which only re quie red arrab gement by author. This long delay meant that Mexican libraries developed their own classifications for their law materials.

True, be gin ning in 1969 and du ring the se ven ties the Li brary of Con gress pu blished she du les for An glo- A me ri can law. Howewer, not un til the eigh ties did sche du les appear for Eu ro pean and La tin Ame ri can law, the first for Ger man law and then for French law, com plemented by the regional classifications for LatinAmerican and European law, KDZ, KG-KH and KJ-KKZ respectively.

Af ter brief re su mes of the sche du les for in ter na tio nal law, subc las JX and ge ne ral law, su class K, the re gio nal clas si fi ca tions are in trodu ced for Eu ro pean and La tin Ame ri can law. The se are com po sed pu rely of ta bles from be gin ning to end, of re gio nal law and then national jurisdictions with their sub di vi sions for lo cal law. The appli ca tion of the se tables are ex plained in re fe ren ce to La tin Ame ri can law. 
Now that the Li brary of Con gress has ela bo ra ted sche du les for the areas of law most im por tant for Mexicanlibraries, the questions of applyng them is in order.

Duran te largotiem poen la Biblio te ca delCongre so de Washington, no había una clasificación propia para el Derecho. En su lugar se usaba los siete puntos de Martell para las obras generales de cada materia que incluía entre ellos un número para esa disciplina. En la colección general de la Bi blio te ca del Con gre so, las obras ju rídi cas es ta ban distri bui das a tra vés de toda la cla si fi ca ción. La Biblio te ca del Derecho, es una colección separada de la Biblioteca del Con gre so, que no re pre sen ta ba ne ce si dad para una cla si ficación como ocurría en la mayoría de las bibliotecas de la materia, dado que el de re cho an gloame ri ca no se de sa rrolla por me dio de lar ga se ries de ca sos y re sul ta ba más efi cien te un orden fundamentado en la secuencia alfabética de los materiales.

El criterio de distribuir las obras de materia jurídica por toda la clasificación que tenía la biblioteca del Congreso, fue inicialmente adoptado por la Dirección Ge ne ral de Bibliotecas de la UNAM y muchas bibliotecas universitarias siguieron su ejemplo. En vista de esta proble mática, la Dirección elaboró algunos esquemas breves de la clase $\mathrm{K}$, para el derechogeneral, de re cho ci vil, de re cho pe nal y derecho procesal con algunas subclase para regiones y países, pues to que eran ra mas no in clui das en la cla si fi ca ción de la Biblioteca del Congreso. Esta práctica resultó útil para las coleccionesjurídicas en un sistemadepartamental donde aparecerían juntas todas las obras de derecho.

Las bi blio te cas con una co lec ción es pe cial en De re cho, integradas a una biblioteca general, no podían seguir la Clasificación del Congreso donde las obras eran esparcidas a través de toda la colección. Requerían tenerlas juntas. Por lo tan to en ins ti tu cio nes como la Bi blio te ca "Fran cis co Javier Clavijero " de la Universidad Iberoamericana (Villoro, 1967; Fernández Galán, 1978), el Instituto de InvestigacionesJurídicas de la UNAM (MorineauIduarte, 1983) y la coordinación de Servicios de Información de la Universidad Autónoma Metropolitana, (Abell B., 19831985), se elaboraron esquemas propios para sus colecciones jurídicas. La clasificación de Los Angeles Country Law Library (1965), sigue la misma práctica, en general, que las bibliotecas mexicanas.

Por cier to, en los años se sen ta y se ten ta em pe za ron a apa recer, paulatinamente, los esquemas para el derecho an- gloamericano, in clui dos en la sub cla se KD para el de re cho británico e ir lan dés (Li brary of Con gress, 1973), la sub clase KE para el derecho canadiense (Library of Congress, 1976) y la subclase KF para el derecho norteamericano (Li brary of Con gress, 1969), sin em bar go, so la men te la segun da edi ción de la sub cla se JX para el de re cho in ter na cional (Library of Congress, 1924) incluyo las adiciones y cambios, hasta 1965 servía para las bibliotecas latinoameri ca nas. Es de cir, fue has ta los años se sen ta en que la cla se K comenzó a desarrollarse sin presentar opción concreta para el enfoque especial del derecho latinoamericano.

A fi na les de la dé ca da pa sa da apa re cióel se gun do es que ma para el derecho en general (Library of Congress, 1977). Esta par te de la cla si fi ca ción era un an ti ci po de la cla si fi cación con raíces romanas que entonces estaban por publicarse.

Hace ape nas cin co años, todo em pe zó a cam biar con la publicación del esquema para el derecho aleman, la clase KK-KKC (Li brary of Con gress, 1982). Más tar de apa re ció el esquema para el derecho latinoamericano , la KG-KH, que incluye América en general, en la subclase KDZ (Library of Con gress, 1984). Ape nas hace dos años la Bi blioteca del Congreso (1985) publicó el esquema para el derecho francés, la KJV-KJW.

El año pa sa do los es que mas cla ve para com ple tar el cua dro del de re cho eu ro peo re gio nal y el de los de más paí ses eu ropeos incluidos España, Ita lia y Por tu gal, la cla se KJ- KKZ, básico para nuestras bibliotecas, vieron la luz en una edición pre li mi nar. Tam bién en una sub cla se de la mis ma apare ció el es que ma del de re cho ro ma no, la KJA, que sir ve de fundamento para los esquemas de Europa Meridional y Amé ri ca La ti na. La cla si fi ca ción del de re cho en las de más ju ris dic ciones del Vie jo Mun do está en pre pa ra ción, la clase KL-KWZ y existen planes para desarrollar el derecho antiguo y los sistemas teocráticos del derecho KB-KC.

El esquema latinoamericano por fin se tiene elaborado, éste ini cia una nue va pau ta. Para las tri ples le tras asig na das a cada país im portan te de la re gión, se uti li zan ta blas ge nera les para va rios paí ses en con jun to con al gu nas ta blas más para temasespecíficos de forma. Esto es una nuevamodali dad. El do cu men to re gio nal eu ro peo y de los paí ses in di- 
vi dua les con sus tri ples le tras si guen una nor ma ini cia da en los esquemas latinoamericanos. Son enteramente nuevos los esquemas que existen para el continente europeo, la KJV-KJW para el de re cho fran cés, la KK- KKC para el dere cho ale mán y el de re cho re gio nal y de los otros paí ses eut ropeos, la KJ-KKZ. Sumados a los nuevos esquemas para elderecholatino americano, la KG-KH.Losclasificadores ten drán que en fren tar lo en el fu tu ro y em pe zar a uti li zar lo. Una exposición parece especialmente urgente debido a que no exis te nin gu na guía para su uti li za ción. Uni ca men te para el derecho ale mán y eu ro peo se cuen ta con una dis cusión so bre las prin ci pa les pau tas que si gue la Bi blio te ca del Congreso para países que se basan en el derecho romano (Goldberg 1981-1987). Esta exposición, para el conocimien to del es que ma, se fun da men ta con ejem plos ilus tra tivos de algunos problemas a que se enfrentarán los clasifica do res en la aplica ción del es que ma para el de re cho latinoamericano en los próximos años.

Para la mejor orientación de los clasificadores, se entrará pri me ro en un re su men ge ne ral de las prin ci pa les sub cla se de derecho, la subclase JX para derecho internacional, la subclase $\mathrm{K}$ para el derecho general y las clases KJ-KKZ para el derecho europeo.

\section{Derecho internacional (Subclase JX)}

Al desarrollarsela clase $\mathrm{K}$, la subclase JX ha sufrido algunas adiciones y cambios. Además, es conveniente revisar sus principales ramas para saber lo que in clu ye y lo que no incluye referente a la clase $\mathrm{K}$ y otras áreas.

JX1-1299 Divisiones generales de forma. Coleccionesgenerales deconvencionesinternacionales referente a relaciones políticas, correspondencia diplomática, casos, reclamaciones, etc. Los tratados constituyen las fuentes principales del derecho internacional.

1305-1599 Relacionesexterioresreferentesalateoría del derecho internacional. Se clasifica la his to riadiplomáticaen las cla ses DyE-F.

4620-4630 La diplomacia. El servicio diplomático. Se refiere a su organización y administración.

4620-4631 el procedimiento en litigios internacionales. Arbitrajes internacional. Organismos internacionales. Es decir, Derecho procesal internacional.

$4620-4632$

la his to ria y teo ría del de re cho in ter na cional. Aquí se clasifican obras generales sobre el derecho internacional público y sobre algunos tópicos específicos.

$4620-4633$ Derecho de guerra.

Las siguientes ramas han sido desarrolladas con mucho más de ta lle en las adi cio nes y cam bios de la Bi blio te ca del Congreso:

JX5401-5486 Responsabilidad internacional. Derecho penal internacional.

4620-4630 Derecho aéreo internacional. Derecho especial internacional.

En un úl ti mo lu gar, la Biblio te ca del Con gre so ha trans feridotodala rama so bre elDe re choin ter na cional privado, antes JX6001-6650, a la cla se K y ha brá más cam bios cuan do se dedique a la alineación de esta subclase con los esquemas regionales que están por terminar.

\section{Derecho en General (Subclase K)}

Este es que ma, publica do en 1977, es el pri me ro de la cla se $\mathrm{K}$ que es de interés para los clasificadores latinoamericanos que uti li zan la Cla si fi ca ción del Con gre so. Aquí se observa, es pe cial men te, con el ru brode de re chocom parado, la estructura general que se encuentra entre los esquemas latinoamericanos. Se subdivide la subclase K entre las siguientes áreas:

K1-195

$4620-4630$

$4620-4631$

$4620-4630$

$4620-4631$

$4620-4632$

$4620-4630$

4620-4631

3150

$4620-4632$

$4620-4633$
Divisiones generales y de forma

Filosofía y teoría del derecho

Derecho comparado

Sus subdivisiones se desarrollan como sigue:

Derecho privado

Derecho civil

Derecho mercantil

Incluye Propiedad Intelectual

Derecho social

Derecho procesal y procesal civil

Derecho público

Derecho constitucional

Derecho administrativo

Con la reglamentación de la industria y comercio: 
4620-4630 Derecho fiscal

4620-4631 Crisis nacionales, Derecho militar

4620-4632 Derecho penal y procesal penal

4620-4630 Derecho internacional privado, es decir, con flic tos de le yes que se cla si fi can en esque masregionales des pués del de re choen general. Esta rama también se subdivide en formas semejantes al derecho privado que ya tra ta moseneldere chocom parado: Temas de derecho civil:

$4620-4630$

$4620-4631$

$4620-4632$

4620-4633

$4620-4634$

$4620-4635$

4620-4636

Personas

Propiedad

Derecho sucesivo

Obligaciones

Derechomercan til. In cluye de re cho marí timo

Derecho social
Derecho procesal civil. Derecho procesal civil internacional

\section{Derecho Europeo (Clase KJ-KKZ)}

El resumen de las clases KJ-KKZ también sirve de pauta para la estructura del derecho latinoamericano. A los esquemas del derecho europeo se insertan los esquemas del derecho francés y alemán en su lugar alfabético.

$\mathrm{KJ}$

Historia del derecho en general. Derecho germánico

KJA Derecho romano

$\mathrm{KJC}$

KJG.KKZ Derecho regional general y comparado organización e integración regionales

Países individuales

Francia, véase KJV-KJW

Alemania, Alemania Occidental. Alemania Oriental, véase KK-KKC

Los es que mas para el de re cho fran cés y el de re cho ale mán son los últimos que se desarrollan para países específicos que sir vie ron de base para la apli ca ción de las cla si fi ca ciones re gio na les. Los de más es que mas como ya se ha he cho en el caso latinoamericano, se elaboran únicamente en base a tablas generales de materia aplicable a regiones y paí ses, y otras que tam bién sir ven para los es ta dos, pro vincias y ciudades de los diferentes países. La Biblioteca del Congresoclasificalahis to ria del de re cho eu ro peo, el de recho germánico y el derecho romano junto con el derecho com para do y re gionalen las sub cla se KJ-KJEse gui das por el derecho de los países individuales en la KJG-KKZ en subcla ses pro pias de triples le tras arre gla das en for ma al fabética.

El derecho de las otras regiones del Viejo Mundo, clase KL-KWZ se gui rán la mis ma pau ta que los otros dos es quemas regionales que se han desarrollado, con tablas para países que uti lizan el de re cho an gloame ri ca no y el de re cho románicorespectivamente. Se han asignado a sus regiones y jurisdicciones estatales la subclase KL para el derecho soviético, la KM-KPZ para el derecho asiático, la KQKTZ para el derecho africano, la KU para el derecho australiano, la KV para el derecho oceánico y la KW para el derecho antártico que integrarán el tercer y último esquema regional.

\section{Derecho Americano y Latinoamericano (Clase $K D Z, K G-K H)$}

En la lista de jurisdicciones y asignaciones de números al principio del esquema latinoamericano se puede apreciar cómo está or gani za da geo gráfica men te estaclase. Se asignan do bles y tri ples le tras a las re gio nes y países den tro del área en cuestión y luego una serie de tablas de materias para re giones, paí ses, provin cias y ciu da des (cuadro1).Entre paréntesis, después de cada área, se indica la tabla de materias que se deben utilizar con letra, es decir, tablas A,B, C, D, E,F y G.

La tabla A se usa para regiones. Por ejemplo: la KDZ 0999, la única subclase de este esquema que geográficamen te in clu ye una re gión fue ra de Améri ca La ti na, se utiliza para el derecho de América y América del Norte en general. Luego con la América Latina en general, KG 3000-3999; para las An til las en ge ne ral, KGJ 0- 999 y para América del Sur en general, KH 0-999; también se aplica la misma tabla A.

Para países con toda una subclase, cada uno asignado con triple letras, se utiliza la tabla B de materias por ejemplo: con Belice, la KGA. Para provincias y estados dentro de cada país importante, por ejemplo con México, la KGF, cada uno con su blo que de nú me ros se re quie re el uso de la tabla D. Para ciudades importantes con bloques de números, se apli ca la ta bla E y paraotrasciudades me nos im portantes la tabla F.

También para Argentina se utiliza la tabla B de materias con sus letras KHA y las otras tablas de ma te rias, la D, E y F ya indicada para México en los casos de estados, ciudades importantes y otras ciudades respectivamente. 
Para algunos países pequeños que tienen asignados solamen te un blo que de nú me ros den tro de una sub cla se de triples le tras, se apli ca la ta bla $\mathrm{C}$ de ma te rias como en el caso de las Antillas Francesas, KGR3000-3499 y la tabla G se utiliza únicamente con la OEA, kdz1100-1199.

Esta es la nueva pauta que la Biblioteca del Congreso ha promovido en América Latina. Para regiones usualmente de dos le tras, se usa una ta bla ge ne ral, para los paí ses de triples le tras se apli ca otra y con es tos, para es ta dos, ciu da des mayores y otras ciudades respectivamente, se asigna para cada ca te go ría una ta bla di fe ren te. Ade más den tro de cada tabla de ma te rias, se hace re fe ren cia a otras ta blas de for ma con que se desarrolla un tema.

\author{
Lite de jurladicsianes y asigaciones de núesres, Cl ase KOZ, XG-KK \\ KD2. 1G-KM \\ LAV OF IXE ARERICAS, LAIIA AHERICA \\ NO2, $\mathbf{X G - K H}$ \\ ano IHE vest InOIES
}

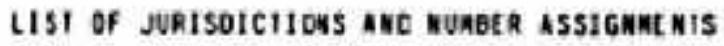

KO2

KG

$0-999$

$3000-3999$

IGA

KCF

0.5999

$6200-5299$

$8300-5399$

$6400-6699$

$6500-6599$

$6600-5599$

6700-5799

$5800-5899$

$6900-6999$

1000-7099
Anerica. North Meerict

Including the entire western Heoisphere

General (table a)

Organitation of Aaerican States (1able 6) Individual countries

etrouda $(1,0)$ i c

Conida, an $\mathrm{kr}$

Greenland (lable $\mathrm{Cl}$

3t. pierce and kigutlan (table C)

United States, 3es KF

latin Aeerica

Including Mexico and Central and South Aeerica

Groeral (Table A)

Organization of Aorrican State1, he K0z1100.

Nexica and Central Aatrica

Gentral (Table A)

Individual countries

Ballite

General (tabla B)

Cities, A.2 (loble F)

British Handuras, wee $\times G a$

Mexito

Geaeral (1ab) B)

States. Foderal Oisteict (1ab) o)

Iquascalientes

Daja california

gajo Californis Sar

Canoecho

Chiopes

Chithuahus

Coahuils

Colioa

Durango

Tederal District, see KGF 7600. 
$7100-2199$

$2200-2299$

7300-7399

$7600-7699$

7300-7399

2600.7699

$9600-9619$

$9620-9639$

$9640-9639$

$9660-9679$

$9680-9699$

9700-9719

9900

20J

0-999

IGA

0.5999

6200.6299

$9600-9619$

9840-9659

$9660-9679$

9800

KCA $3000-3499$

Kn

0.999

KMA
6200.6298
6300.6399
$6600-6499$
6500-6599
$6600-6699$
6700.6799
6800.6399
6900.6999

$7000-7098$

7200.1289

7300.7399

7400-7499

$7500-7699$

7700- 7799

$7300-7898$

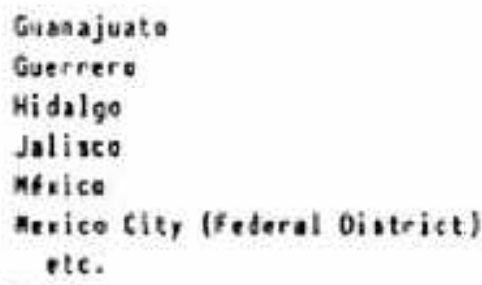

Mest Indies. Caribbean arta

General (lab!e A)

Individual islands and graups of islands

cubs

Genera] [loble a)

Provinces (table 0 )

Canagler

Cities

Havans, see KGW7700.

Hol quin trable $E$ )

Sante Cloro (table E)

Santiago de Cubs (table E)

Other, A-2 (table f)

French rest Indiea (General) (1able C)

South Aoerica

General (Table a)

Individual countries

Argentins

General (table B)

Provines. Foderal oistriet. Motional lerritory (tablo o)

buenos aires (Federal Oistrict)

Outnos Alres (Prouince)

Catasares

Chace (Presidente Perba)

Chubut

csedoba

Corrientes

intre Rises

Eva Per6n, Iee KHA7zO0.

Federal Oistrict, set K\$ab200*

fornosa

Jujur

Ia Pama (Eva Perán)

Ia Rioja

Męndoza

Mistiones

Maveutn

Petsidente persn, see knabso0. 


\begin{tabular}{|c|c|}
\hline $\begin{array}{l}7900-7999 \\
0000-0099\end{array}$ & $\begin{array}{l}\text { Afo negeo } \\
\text { salte }\end{array}$ \\
\hline $0100-19$ & sin Jaen \\
\hline 0200-0299 & San teils \\
\hline 0300-0399 & Sonto Cruz \\
\hline $6400-0699$ & Santo te \\
\hline essoo-essos & Santiago dol Estere \\
\hline $0600-6699$ & Vierra del ruage \\
\hline $0100-079$ & Tucuedn \\
\hline & sitins \\
\hline $9600-9619$ & 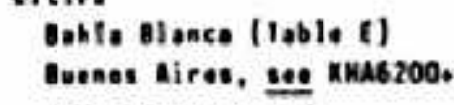 \\
\hline $9640-9659$ & Cordabs (Iable E) \\
\hline $9660-9619$ & Lo Plate (Toble C) \\
\hline $9600-9699$ & Mendoza (rable $t$ ) \\
\hline ข700-9718 & Parans (Tabl, E) \\
\hline $9720-9139$ & Mosario (1oble E) \\
\hline $9740-9753$ & Sante fo (Toble E) \\
\hline $9760-9779$ & fueuadn (10biet) \\
\hline 9000 & Dether, $a-z$ (nable f) \\
\hline
\end{tabular}

Con las letrasescogidas para la re gión o país per ti nen te, se pasa a la tabla de materias indicadas. Por ejemplo, para América Latina en general tenemos las letras y bloque de nú me ros KG 0-999 (cua dro 1)n con la in di ca ción de usar la tabla A (cuadro 2). Obsérvese que algunos temas van seguidos con números romanos entre paréntesis, (I) (II) y (VII) Es tos nú me ros in di can que se tie ne que usar con es tos temas algunas tabla de divisiones de forma. En el caso de AméricaLatina se uti li za prin ci pal men te la ta bla I para temas asig na dos con nú me ros en te ros y la ta bla II Para ma terias asig na das con nú me ro de cut ter. La ta bla VII se uti li za con organismos regionales que no sea la OEA. En esta exposición veremos únicamente la tabla I.

\section{América Latina (General) (Tabla A) \\ Con tabla I (regional -1 número)}

KG432 El derecho latinoamericano del trabajo /Mariano R. Tissenbaum.. [et al.]-

.A63D4 México: UNAM, 1974

KG574 Veinte años de evolución de los derechos humanos: seminario
.A6 internacional patrocinado por la Secretaría de Relaciones Exteriores de

1974 México y la Comisión Interamericana de Derechos Humanos /

Colaboradores: Cassin...[et al.]-México: UNAM, 1974

KG735 La dimensión jurídica de la integración, América Latina /INTAL.-

.D5 —Buenos Aire: INTAL, 1973

Obsérvese que con el de re cho la bo ral ge ne ral se gún el primer ejem plo, se uti li za el nú me ro 432 con base en la ta bla I. (cua dros 2y3). Como en este caso, se tra ta de una co lec ción de es cri tos de va rios au to res, agre ga mos al nú me ro de clasificación KG432 los nú me ros de cla sifica ción KG432los números de Cutter .A63 y luego D4 por el asiento. Para el siguien te ejemplo sobre de re chos hu manos, te ne mos el núme ro 574 se gui do de la ta bla I in di ca en tre pa rén te sis (cuadro 2). Por ser un congreso se agrega a la clasificación, KG574, el Cutter .A6 y la fecha 1974(cuadro 3). Al tema sobre la "Integración Económica Latinoamericana", KG735 General (I) (cuadro 2), se agrega únicamente .D5 por ser obra general (cuadro 3 ). 
CUADRO $?$

102, $16.2 \mathrm{k}$

40

1.92

133

4.35

436

4.16

$\$ 40$

574

576

578

578

500

335

218

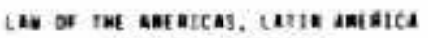
aD tur ue it tabtes

Lase a

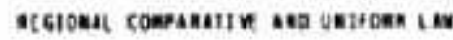

norke on the low of. ond treation betwern, twe or ose soustriet in the labe cegion

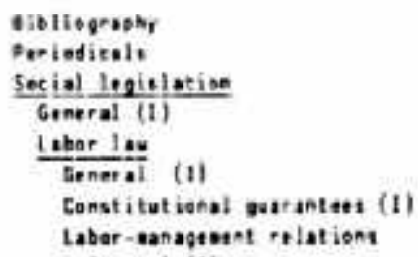


CUROMO 3

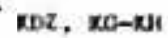

. $A 2 A-2$

$\rightarrow 25$

, 3 3 339

A3SA -2

,$A 37 k-2$

A4tease|

. ASZ $\mathrm{A}-\mathrm{Z}$

.ASSA=

$. A 57 \mathrm{~A}-2$

ASAR-Z

. 16

$.463 \mathrm{~A}-2$

$.164 \lambda-2$

$.465 a-2$

$, 1)-25$

$.27 \mathrm{~A}-2$

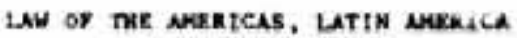

AND THE URST INDIES

rost olvisions

TAm: :

(Retionel - i nubber)

\section{Mibltegraptr}

Fertodicalis

Clate bere pertodleale cansioting prtastidy of informative aeteriad (aeveletiers, bulletine, ete.) relating to articular oubject

For perlodicals conaieting peodoelnant ly of legal articlea, ragardiase of eubject

atcer and furiodiction, ere $\mathrm{K} 1+$

Honographic eezice

letergovernental congrases and conferencel

Far ether congresues, wetinge, etc., sec , 46

Ceseral

Seriale

Honegrapha. Iy date

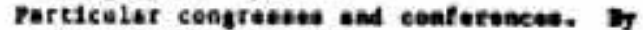
nawe of the congrees

arreeged elphabetically, by eane of euccensive Cucser natbere

Ueder euch:

-xi15-169 Seriala

- xN2 Nonegrepha. By dote

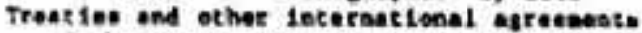

ró treat les aot lifested to countries of

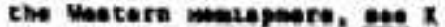

Collections

Includint efther mithlateral or bileteral trastios or both

Caneral

freaties of a pacticuler country. By coustry end dete

Clase hare vorke Ifales to treatias - wonk countefet withis the toglon

tholestateral treation

Decleibat. Mdainfstrative rulinge

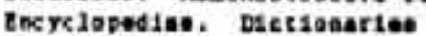

Fors booke

7earbeoke

Clase here publieatione tooved anuelif. vunarizias eveate, otaktitico, ete.. of the year. For ocher publtestiens eppearing yearly, wee.Als

Congrebsea. By dote of congreos

Por Interneverneental concrueses and conferences, wet. A2t

Collected worke (nonearial)

several suthore

Individual authore

Carbeoks, rebdiaga

Ceneral vorke

Adreaseb, eoere, Lectures

Including ingle verye, collected eseaye

of several suthere, Fetechriftea, ete. 
Para países asignados con triples letras por ejemplo en los ca sos de Mé xi co y Ar gen ti na (cua dro 1), se uti li za la ta bla B (cua dro 4). Se si gue con al gu nos ca sos con cre tos so bre el modo de manejar esta tabla de materias, primero con una tabla interna y después con tabla de divisiones de forma:

\section{Argentina y México (Tabla B) Con una tabla interior}

KHA2914 Argentina

1853 [Constitución (1953)]

.A315 Constitution of Argentina, 1953, as amended -Washington: Pan American Union, 1968

\section{Con tabla III (países -10 números)}

\section{KGF4624 México}

1966 Legislación del impuesto sobre la renta /Ernesto Flores Zavala y

Torres Torija.-México: Ediciones Flores Zavala, 1966

KGF4629 Escorza Ledesma, Juan

.E8 Tratado práctico del impuesto sobre la renta. - México: Cárdenas, 1971

Con tabla IV (países, estados, provincias, etc. 1 número)

\section{KGF4796 México}

.A31976 Ley general del timbre de 1976.-México:

A7

Trillas , 1976

1976

Para Argen ti na se uti li zan las le tras KHA (cua dro 1) como ya se visto y para constituciones individuales, el número 2914 con que se utiliza una pequeña tabla interna (cuadro 4). Para la tra duc ción al in glés de la Cons ti tu ción Ar gen tina, materia del primer ejemplo, se agrega después de la fecha de la Constitución 1853, el Cutter .A3 por ser una edición en idioma extranjero y luego el Cutter del idioma, AZ, 15 para el inglés.

Cuando se clasifican materiales por países (cuadro 1) normalmente el procedimiento indica el uso de la tabla $\mathrm{B}$, de ma te rias (cua dro 4), con que se uti li zan ade más la ta bla de forma III (10 números), la tabla IV (1 número) y la tabla V(i nú me ro de Cut ter). Por ejem plo, para im pues tos me xicanos sobre la renta, KGF4621-4630 (cuadro 4) se usa la ta bla III de for ma (cua dro 5) que para co lec cio nes de es tatutos como monografía se da el número 4, o KGF4624 al cual se agrega la fecha. Para una obra general, el tercer ejem plo, se es co ge el nú me ro 9, ó KGF4629 a que se agrega el Cutter del autor,.E8 cuadro 5).

Para ley general de timbre, la tabla $\mathrm{B}$ nos da el número 4796 y la ta bla IV de un nú me ro en te ro (cua dro 6) nos da el resto de la clasificación.

Para leyes individuales el Cutter es .A3, se agrega como parte del mismo la fecha de su promulgación, .A31976. Lue go para tex tos mo no grá fi cos de la ley se adi cio na.A7 y la fecha.

Ahora veremos cómo se utiliza la tabla de forma $\mathrm{V}$ que se es co ge cuan do tie ne úni ca men te un nú me ro de Cut ter de la tabla B.

\section{Con tabla V (países, estados, provincias, etc. - Cutter)}

\author{
KHA895 Banchs, Irineo Ernesto \\ .B34B3 Contratos detrabajobancarioydeseguros \\ / Irineo Ernesto \\ Banchs, - Buenos Aires : E.D.I.A.R., 1967
}

1. Empleados bancarios - Argentina 


\section{KGF1962 México}

.B8A3 Ley federal de los trabajadoresalservicio del estado:

Reglamen tariadel apartado B del Art. 123 cons ti tu cional, concordada con la Ley fe deraldel tra bajo, Ley de Ins ti tu to de Seguridad y Servicios Sociales de los Trabajadores del Estado y disposiciones conexas.-4a . Ed. -México: Andrade, 1973

1

Burócratas

Para el siguiente ejemplo argentino (cuadro 1), se tiene el número KHA895, A-Z, para contratos laborales con la in- di ca ción del uso de la ta bla $\mathrm{V}$ para nú me ros de Cut ter (cuadro 7) y por ser obra ge ne ral se agre ga al nú me ro de Cut ter de empleados bancarios, .B34, el Cutter de autor. Por casualidad se en cuen tra con el De re cho Laboral el nú me ro de Cutter para empleados bancarios y por motivos de uniformidad parece completamente razonable utilizarlo en este caso. Otra ley mexicana (cuadro 1) sobre el derecho laboral, KGJ1962 para tra ba ja do res al ser vi cio del es ta do o bur rócratas (cuadro 4) también se asigna la tabla $\mathrm{V}$ para números de Cutter para burócratas. Por no tener el Cutter para burócratas, asignamos el número .B8 según la tabla 3 de au to res y el nú me ro.A3 para tex tos mo no grá fí cos de la ley que se agrega la fecha (cuadro 7).

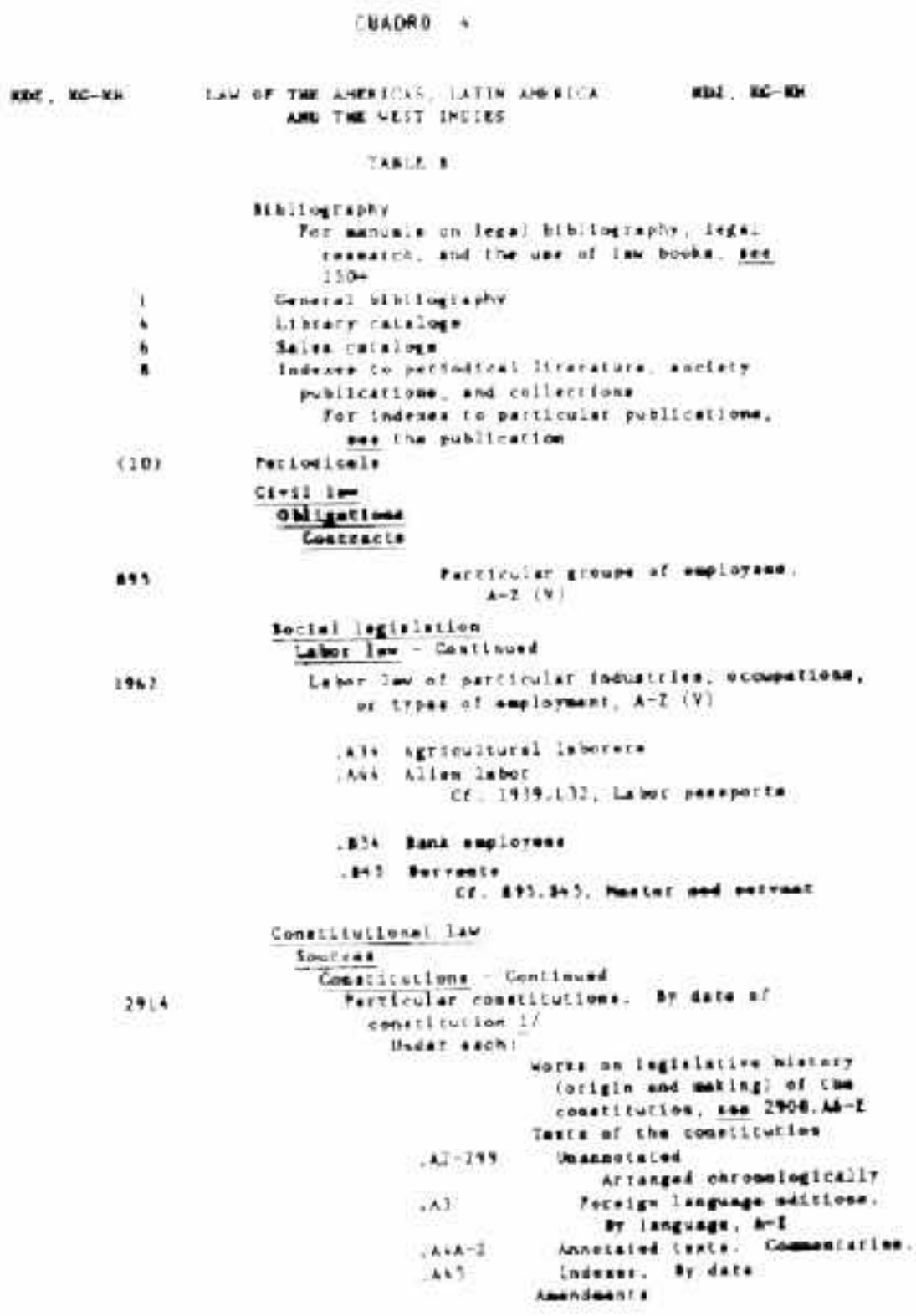




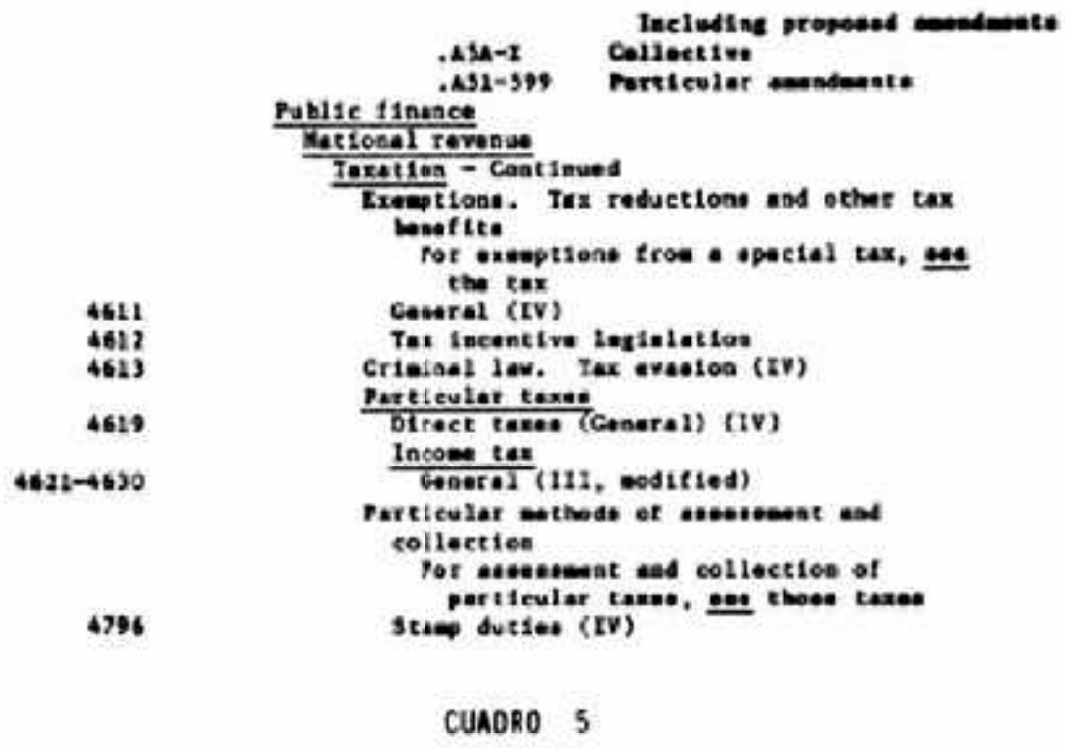

ex, $\mathbf{E C}-\mathbf{E H}$

Law of Dit neracas, Latis anEaICa NW THR WRST INDies

ED2. KG-WR

PONS DIVISIOWS

rasu it:

(Countries - 10 nuabera)

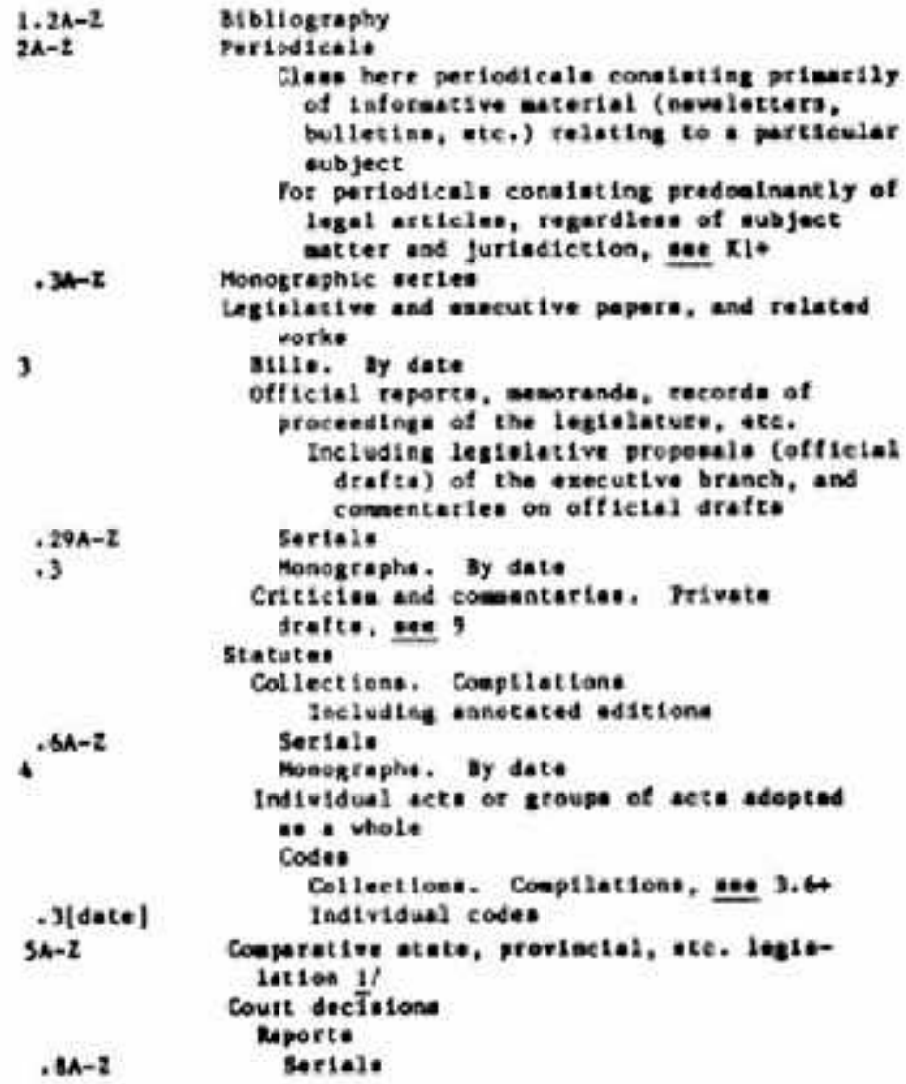




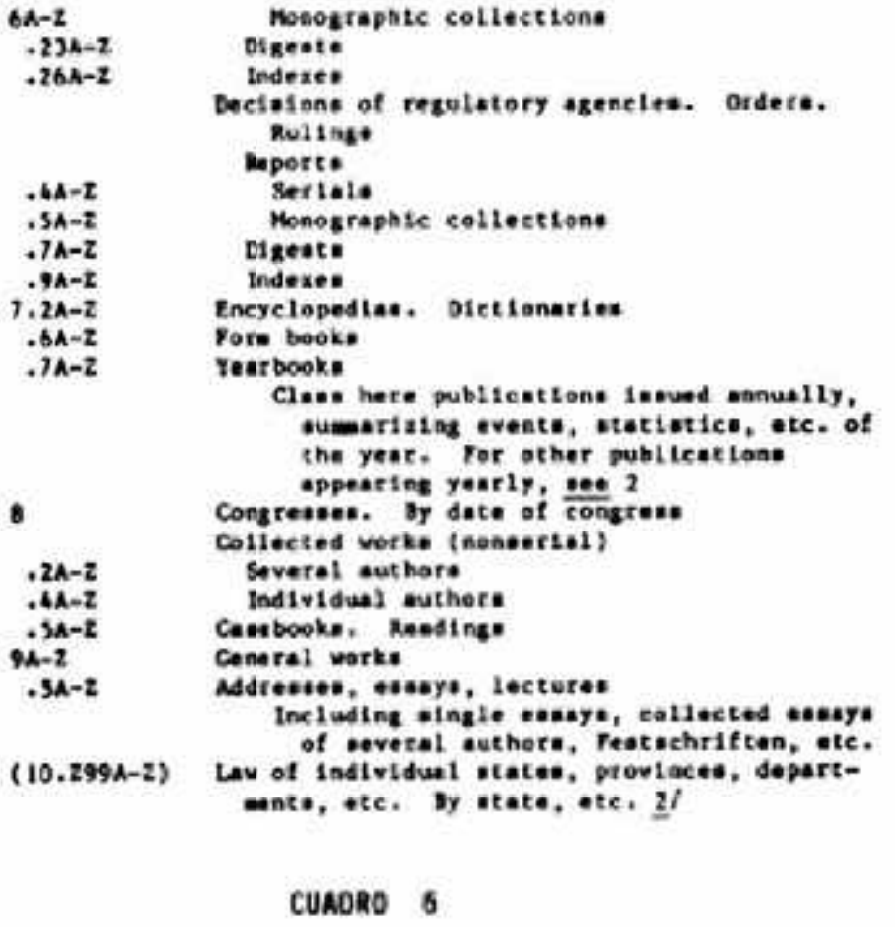

w2), ש-w

LW OF me narteas, LATIM NRRICA AND TRE UEST INDTES

$\mathbf{K D z}, \mathrm{KC}-\mathbf{K C H}$

Fon Divistons

TABY :V

(Cowntries, states, provinese, ete, - 1 mumber)

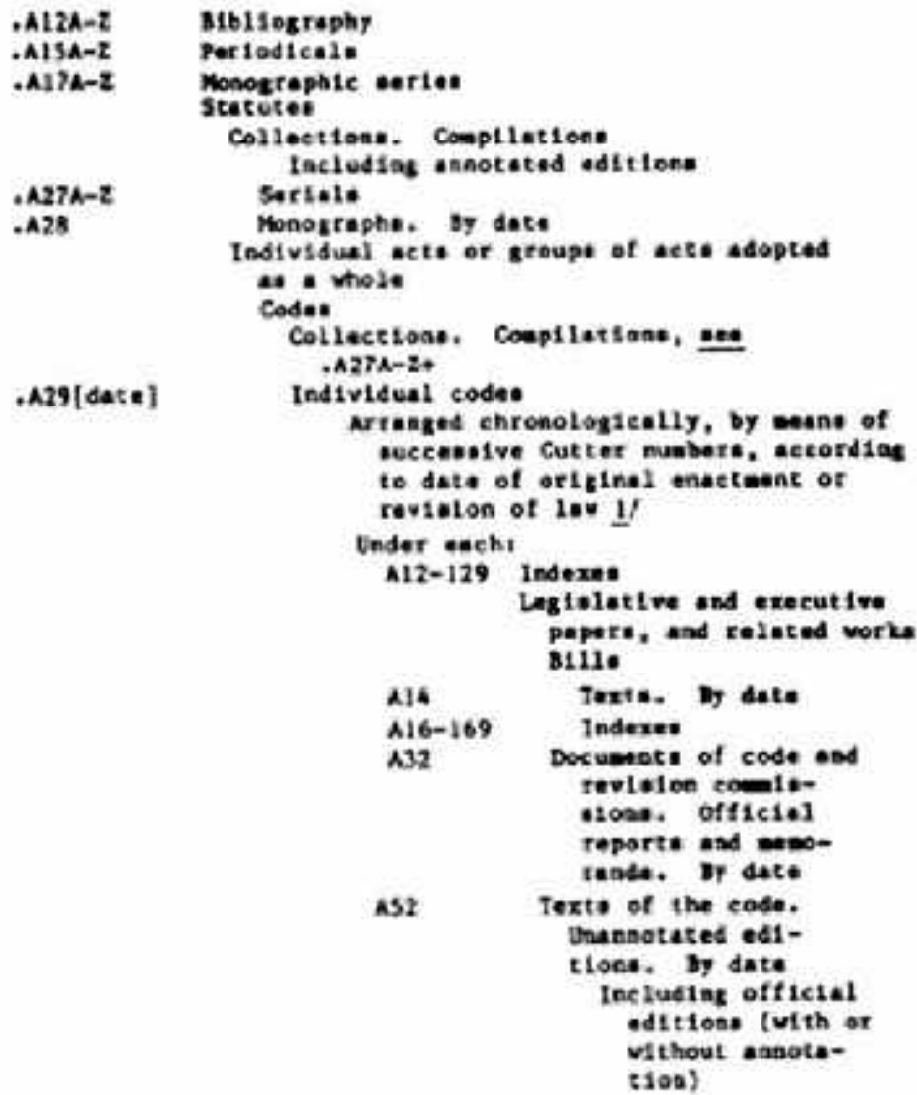




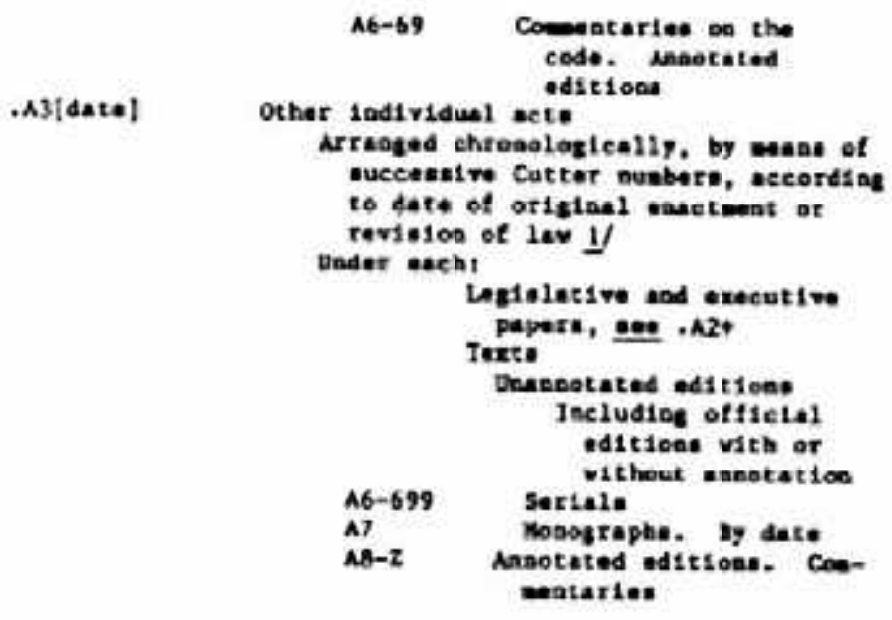

CUADคO 7

KDX, KCF-KH

LAM OT DIE NIRICAS, LATIK NENTCA ND ne vest INDIES

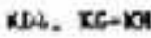

rony DtVisions

TAMLX $\mathrm{V}$

(Countries, atetes, provinces, otc. - Cutter nuaber)

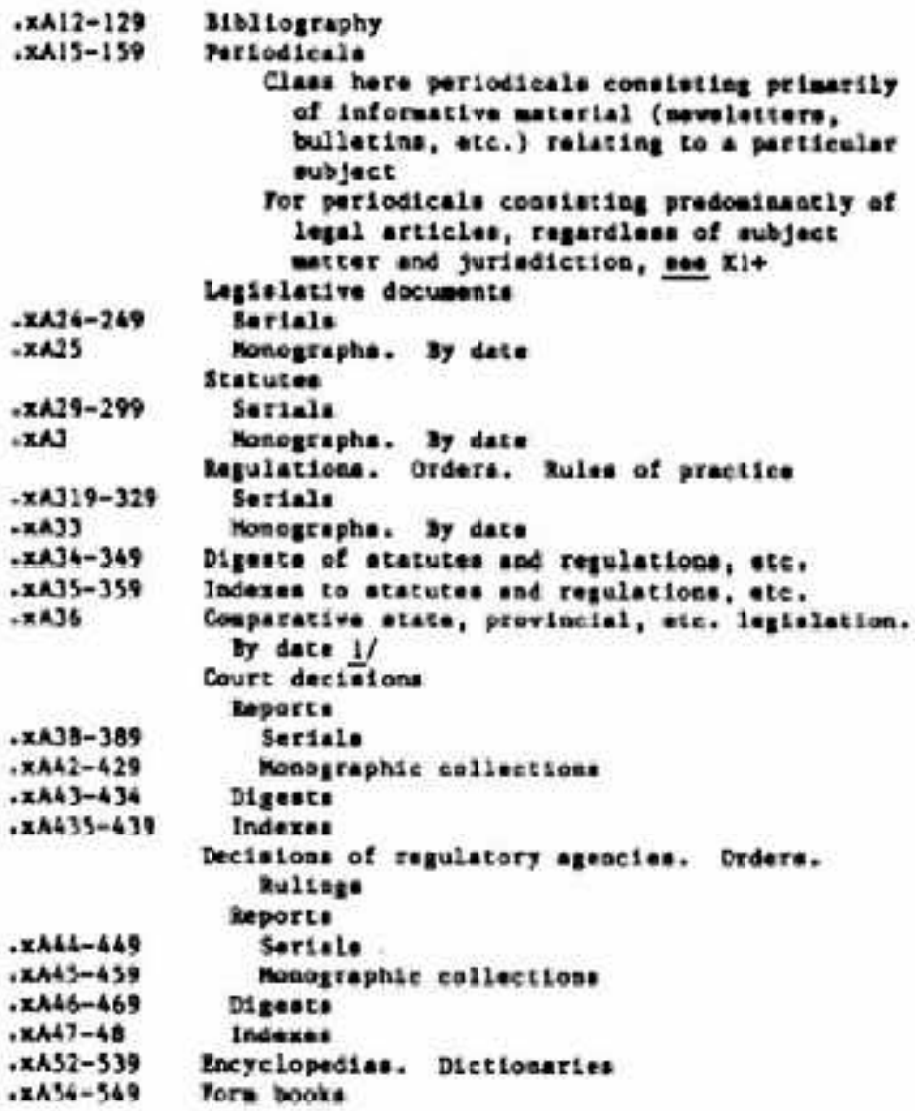




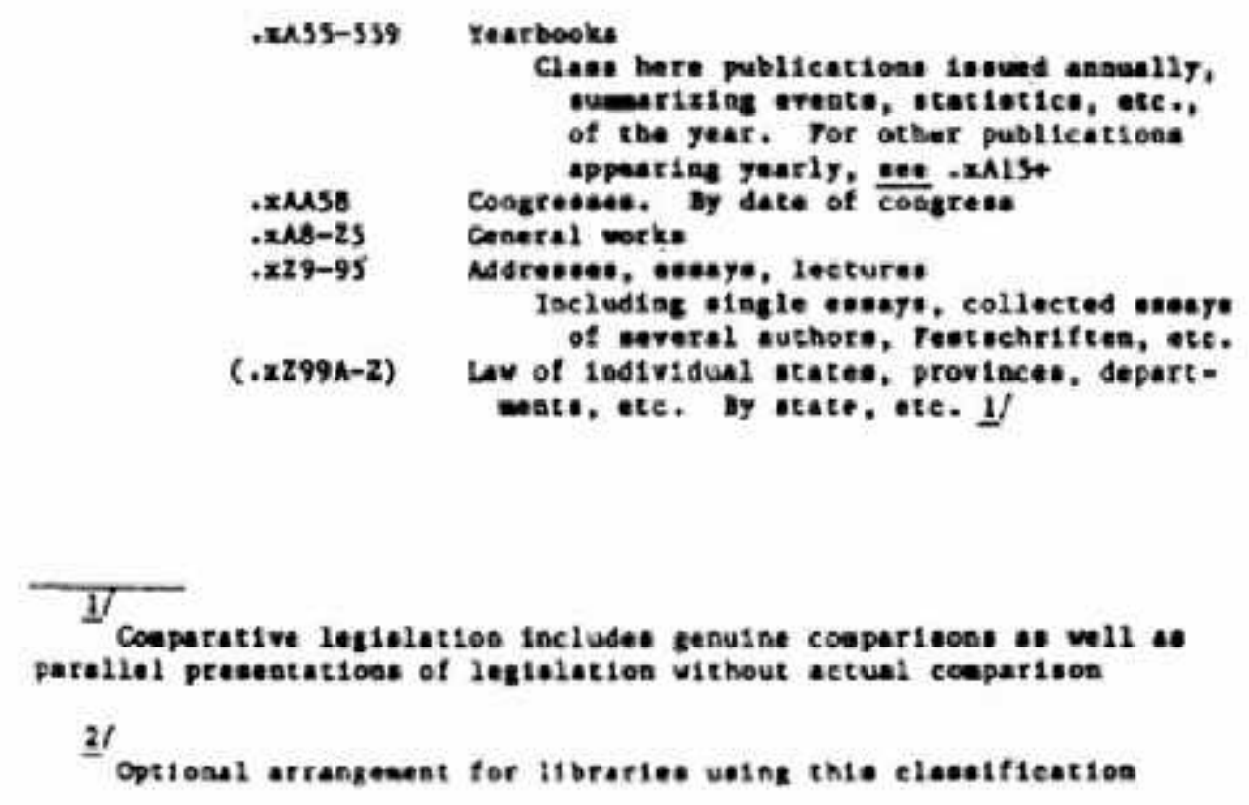

Aho ra se ana li za rán bre ve men te unos ejem plos so bre la tabla D para Estados y en el caso de México va incluido el Distrito Federal, entre los estados con la clasificación KGF7600-7699 (cuadro 1).

Con tabla V (países, estados, provincias, etc. - 1 número de Cutter)

México (D.F.) (Tabla D)

Con tabla IV (países, estados, provincias, etc. -

\section{1 número)}

\section{KGF7611 México (D.F.9}

.A29193 Nue vo có di go ci vil para el Dis tri to y te rritorios federales / anotado y

A52 concordado por Manuel Andrade. $-14^{\mathrm{a}}$ ed. - México, Andrade, 1976-

1976

KGF7611 Muñoz, Luis

.A291932 Comentarios al Código civil / Luis Muñoz, Salvador Castro Zavaleta. -

A66
KGF7672.25 México (D.F.)

.T7A33 Reglamento de tránsito del Distrito Federal. - México: Andrade, 1976

1976

Luego de la tabla D (cuadro 8) para el primer ejemplo sobre de re cho ci vil, se se lec cio na el nú me ro 11, y se usa la tabla IV para números enteros (cuadro 6). Se escoge el Cutter .A29 y se agrega 1932 como fecha de su promulgación, .A291932. Además se agrega A52 por textos del código y la fecha de la edición. se considera que los comentarios son mínimos y subordinados enteramente al texto. Pues, un comentario al código civil trae primero el Cutter .A291932 para te ner la in for ma ción so bre la mis ma edi ción del có di go en un solo lu gar, pero lue go se agre ga el Cutter sobrecomentarista, .A66, se gun do dí gi to por la primera letra del apellido Muñoz según la tabla $\mathrm{V}$ de autores para tener en orden alfabético todos los comentaristas. 


\author{
CUAORO B \\ rat. $10-104$ \\ LAN OF THE NMERICAS, LATIN ANERICA \\ AND THE WEST INDIES \\ EDZ, TC-FH \\ TABLE D \\ STATES. PROVINCES. TERRITORIES $1 /$ \\ tacluding departaent. Federal divtricte. \\ and other flrot order polltical and \\ adedntetrat ive oubsiviosone \\ 1 Mbllography \\ (.2) Periodicale \\ See note under (10) in Table \\ 11 \\ Civil 1ev \\ Ceneral (IV) \\ Rerulation of Indunery, trade, and conrea. \\ Decupetional low - Continued \\ 72 \\ Traneportation and colinication \\ Cenerel (IV) \\ .15 \\ Loed traffic. Autometive tranepertation \\ General (IV) \\ Nator vahicle $1 \mathrm{men}$ \\ General (IV) \\ .2 \\ spectal copica, $A-Z$ (V) \\ ,T7 Traffic regulationa \\ tacluding enforcentent and trafflc \\ rialation:
}

\footnotetext{
I

5ee pp. 3-21 for l1et of etetes, provinces, etc, and nuebere eael rned

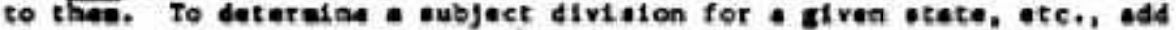
the auber or numbere in the table for the oubject to the buole number for the otete, etc. for example, tor election lev la Tierra del ruego, Argatian, add 38.8 is table to beile aubar for Tiorra del Puago, wiss 500 . to enke wirs 338.8
} 
El último ejemplo de la ta bla D (cua dro 8) se re fie re a tó picos es pe cia les de le yes de trans por te au to mo triz con que se uti li za la ta bla $\mathrm{V}$ por ser nú me ro de Cut ter, .T7 para este reglamento de tránsito. Por ser reglamento monográfico, se agrega un segundo Cutter, .A33 y la fecha.

Se tiene ya casi todo los esquemas que se requieren para clasificar el derecho con raíces romanas. Con la elaboración del derechoeuropeo re gio nal, KJ-KJF in clui do el derecho romano, en KJA con las subclase de signadas parael derecho español, italiano y portugués de la clase KK, se tiene casi todo lo que se requiere para clasificar los libros de raíces romanas según los esquemas de la biblioteca del Congreso.

El cua dro ya es muy di fe ren te a lo que era ape nas hace unos cuatro años. Prácticamente nada aparecido. Sin embargo, en es tos años ha cam bia do el pa no ra ma por com ple to y tene mos casi to dos los es que mas que se ne ce si tan. En ton ces cabe preguntar: ¿qué quieren hacer los bibliotecarios con casi to dos los es que mas que más se ne ce si tan de la Cla si ficación del Congreso que está a su alcance?

Roberto Abell B.

\section{Bibliografía citada}

ABELL B., Roberto. Clasificación de derecho de América Latina, KG-KH [por]

Rober to AbellB., JoséPe rei raZa pa ta. Méxi co, Dep to. de De re cho, Co or di na ción de Servi cios de In for ma ción, Di visión de Ciencias Sociales y Humanidades, Universidad Autónoma Metropolitana, [1983]

- - - . Clasificaciónde derechode EuropaOccidental, KJ [por] Rober to Abell B., JoséPe rei raZa pa ta. Mé xi co, Dep to. deDere cho, Coor dinación de Servi cios de Infor ma ción, Di vi sión deCien cias Sociales y Hu ma ni da des, Uni versidad Autónoma Metropolitana, Unidad Azcapozalco, 1985 (Reporte de investigación, 140)

FERNANDEZ GALAN, María Elena. Derecho; ampliación al esquema K de la clasificación de la Biblioteca del Congre so elabo radoparalascolec ciones de la Univer sidadIberoamericana. México, Universidad Ibe ro americana, 1978

GOLDBERG, Jolande E. "Li brary of Con gress clas si fi ca tion sys tem for Ger man law: a new approach". Law library journal, v.74, no.3 (Summer 1981), p.p. 619-631

LIBRARY OFCONGRES. SubjectCa ta loging Di vi sion “JX, In terna cionalla, Fo reign re la tions, diplomacy, internationalarbitration"en Classification, classJ,Politicalscience. 2daed. Wa shing ton, Li brary of Con gress, 1924

- - -. Subject Cataloging Division. Classification, class K, subclass KF: Law of the United Stated. Prelim. ed. Washington, Library of Congress, 1969

- - - . Subject Cataloging Division. Classification, class K, subclass KD: Law of the United Kingdom and Ireland. Washington, Library of Congress, 1973

- - - . SubjectCataloging Division. Clas sifica tion, class K, sub class KE: Law of the Ca na da. Wa shing ton, Library of Congress, 1976

- - - . SubjectCataloging Division. Clas sification, class K, subclass K: Law (General) Wa shing ton, Li brary of Congress, 1977

- - - . SubjectCataloging Division. Classification, class KK-KKC: Law oF Germany. Washington, Li brary of Congress, 1982 
- - - . Subject CatalogingDivision. Classification, class KDZ, KG-KH: Law of the American, Latin Ame ricaand the West Indies. Washington, Library of Congress, 1984

- - - . Su bject Ca ta lo ging Di vi sion.Clas sifica tion, class KJV, KJW: Law of the France. Wa shing ton, Li brary of Congress, 1985

-- - . Su bject Ca ta lo ging Di vi sion. Law of Eu rope [subclass KJ-KKZ]. Working draft. [Wa shing ton] Sub ject, Ca ta loging Division, Library of Congress, 1986

LOS ANGELES COUNTY LAW LIBRARY. Class K-Law Rev. Ed. Los Angeles, Calif., 1965

MORINEAU IDUARTE, Marta. Sistema de Clasificación de la Biblioteca del InstitutodeInvestigaciones Jurídicas Por Marta Morineau Iduarte, Héctor Dávalos Martínez. México, UNAM, 1983 (Serie E. Varios, no. 26)

UNAM. Dirección General de Bibliotecas. Clasificación de derecho. México, UNAM, 195?

VILLORO, Miguel. Derecho “K”. México, Universidad Iberoamericana, 1967 